\title{
Estimation of Heterogeneous Treatment Effects from Randomized Experiments, with Application to the Optimal Planning of the Get-Out-the-Vote Campaign
}

\author{
Kosuke Imai \\ Department of Politics, Princeton University, Princeton, NJ 08544 \\ e-mail: kimai@princeton.edu (corresponding author) \\ Aaron Strauss \\ The Mellman Group, 1023 31st St NW, 5th Floor, Washington, DC 20007 \\ e-mail: aaronbs@gmail.com
}

\begin{abstract}
Although a growing number of political scientists are conducting randomized experiments, many of them only report the average treatment effects and do not systematically explore the variation in treatment effects across subpopulations. This is unfortunate from a scientific point of view because heterogeneous treatment effects can provide additional substantive insights. This current state of affairs is also problematic from a policy makers' perspective since such studies do not identify subgroups for which treatments are effective. In this paper, we propose a formal two-step framework that first identifies heterogeneous treatment effects from a randomized experiment and then uses this information to derive an optimal policy about which treatment should be given to whom. Our proposed method avoids the risk of false discoveries that are likely in post hoc subgroup analysis routinely conducted in the discipline. We discuss our methodology in the context of getout-the-vote randomized field experiments and show how the proposed two-step framework can be applied in real-world settings.
\end{abstract}

\section{Introduction}

Over the last decade, political scientists have shown renewed interests in randomized experiments that are conducted in the field as well as in the laboratory (Druckman et al. 2006; Morton and Williams 2010). Despite the accumulating empirical evidence through these experiments, political science articles, which report the results of randomized experiments, have a strong tendency to focus on the statistical significance of the estimated overall average treatment effect (ATE) of each treatment rather than systematically study the variation in treatment effects across subpopulations. In addition, many researchers implicitly assume the constant additive treatment effect across units when employing regression models for statistical analysis. This leads to the standard practice of reporting a single estimate (or at most a small number of estimates) summarizing the efficacy of each treatment.

This current state of affairs is unfortunate for both academic and policy-making perspectives. The heterogeneity of treatment effects, if successfully identified, can provide additional insights for the validity of theories under consideration. Moreover, policy makers can take advantage of such information to identify subgroups for which treatments are effective, thereby enabling them to derive an optimal policy of which treatment should be given to whom. Recognizing this problem, a growing number of methodologists are beginning to propose various statistical methods that enable the estimation of heterogeneous treatment effects from randomized experiments (e.g., Horiuchi, Imai, and Taniguchi 2007; Feller and Holmes 2009; Nickerson and Arceneaux 2009; Green and Kern 2010b). This is the literature to which we contribute.

Authors' note: The first version of this paper was circulated in December 2008 under the title of "Planning the Optimal Get-out-thevote Campaign." We thank useful comments from seminar participants at Columbia University and the University of Wisconsin, Madison, as well as three anonymous reviewers and the editor. Supplementary materials for this article are available on the Political Analysis Web site. 
Specifically, we propose a formal two-step framework that first estimates heterogeneous treatment effects from a randomized experiment and then uses this information to derive an optimal policy. Regarding the first step of estimation, we emphasize that any attempt to identify treatment effect heterogeneity needs to address an important and well-known statistical problem of post hoc subgroup analysis in a principled manner. Namely, the subgroup analysis problem refers to the fact that if subgroups are formed after the experiment is conducted in an ad hoc manner, such an analysis runs a risk of finding statistically significant results when no true relationship exists (e.g., Pocock et al. 2002). We show that cross-validation can be used to avoid overfitting of a statistical model and help obtain accurate estimates of heterogeneous treatment effects.

For the second step of optimal policy planning, we develop a decision theoretic framework where given the information about treatment effect heterogeneity obtained from a randomized field experiment, a policy planner optimally chooses, subject to the budget constraint, a treatment from a set of multiple possible treatments for each unit. This framework is based on the recently developed literature about treatment choice (e.g., Manski 2005), but it differs from this literature by adopting a Bayesian approach rather than a frequentist approach based on the maximin or minimax regret criteria (see Dehejia 2005, for an exception). According to the Bayesian criteria, a planner starts with a prior belief about the effectiveness of each treatment under consideration for individual units with different characteristics and then updates this belief based on the available experimental data. Thus, our framework connects the two key concepts, treatment effect heterogeneity and cost-effectiveness, that are currently missing from much of the literature.

To illustrate how our framework can be applied in practice, we discuss our methodology in the context of get-out-the-vote (GOTV) randomized field experiments. Building on the previous work from more than a half century ago (e.g., Gosnell 1927; Hartmann 1936; Eldersveld 1956), many researchers developed creative ways to conduct large-scale field experiments to study voter turnout in real electoral environment (see Gerber and Green 2000; Gerber, Green, and Shachar 2003; Nickerson 2008, among many others). Given the high degree of internal and external validity, the empirical findings of these studies have a potential to significantly impact the practice of GOTV campaigns in the real world (Green and Gerber 2008).

We first show how to estimate heterogeneous effects of various mobilization techniques from randomized field experiments. In particular, we propose a new variable selection algorithm and use it in combination with nonparametric methods and cross-validation procedures in order to avoid the overfitting of statistical models. We then demonstrate how GOTV campaign planners, both partisan and nonpartisan (or academic researchers who wish to inform the planners through their experimental research), can best utilize the results of field experiments when planning their own mobilization campaigns. In the proposed Bayesian decision theoretic framework, a nonpartisan planner maximizes the (posterior) expected turnout among a target population of voters subject to a budget constraint, whereas the objective function of a partisan planner is the (posterior) probability that the party's own candidate wins the election. Under this setting, we show that the standard linear programming algorithm can be used to derive the optimal GOTV campaign strategy.

Finally, we empirically evaluate the effectiveness of the proposed methodology by applying it to three existing field experiments evaluating the efficacy of nonpartisan text messages to mobilize voters, the impact of social pressure on turnout, and a partisan mobilization campaign. In multiple cases, we find that the resulting optimal campaign strategy is more than twice as cost-effective than a naive strategy.

\section{The Formal Framework of GOTV Campaign Planning}

In this section, we follow the literature on treatment choice (e.g., Manski 2005) and formalize the problem of deriving the optimal nonpartisan GOTV campaign strategy from experimental data as a statistical decision problem. We extend this framework to the optimal partisan persuasion and GOTV campaign in Section 4. Rather than following the frequentist approach that is dominant in the treatment choice literature, we adopt the Bayesian statistical decision theory (Berger 1985). We assume that a GOTV campaign planner learns about the effectiveness of various mobilization methods for different voters by 


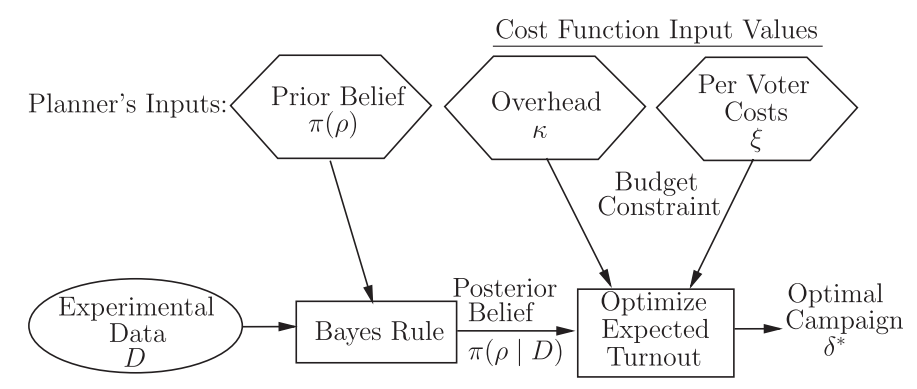

Fig. 1 An overview of the Bayesian optimal campaign planning process. Inputs over which the planner has direct control are represented by hexagons and are (1) the prior belief about the effects of various mobilization strategies on different voters, $\pi(\rho)$, (2) the overhead costs of each mobilization method, $\kappa$, and (3) the cost per voter for each strategy, $\xi$. Data from randomized field experiments, $D$, are represented by the oval. These data and the planner's prior distribution are combined via Bayes's rule to produce a posterior belief about the effects of mobilization strategies $\pi(\rho \mid D)$. Finally, our proposed optimization method uses this posterior belief and the exogenous costs $\xi$ to find the optimal campaign strategy $\delta^{*}$ for the planner.

analyzing randomized field experiments. The planner then maximizes the posterior expected turnout among a target population of voters subject to the budget constraint. ${ }^{1}$

\subsection{Bayesian Optimal Campaign Planning at a Glance}

We briefly summarize the proposed Bayesian decision theoretic framework before we give its formal justification. Clearly, this framework is applicable beyond GOTV campaigns, but for the sake of clarity, we describe it in the context of planning an optimal campaign. Generalization to other situations can be done by replacing mobilization techniques with other treatments of interest (e.g., medical treatments) and voters with other types of subjects (e.g., patients).

Figure 1 depicts the process by which a planner arrives at the optimal strategy. The planner must determine the costs of each mobilization strategy (both overhead and per voter) as well as the prior belief about their effects on voters with different characteristics. Since the cost function inputs are often exogenously determined (e.g., the cost of postage and phone calls), the planner's only meaningful decision might be determining a prior belief. In many cases, the planner might use a diffuse prior centered around a belief that there is no a priori difference in effects of a mobilization method across different voters (but see Nickerson and Arceneaux 2009). This type of prior is especially appropriate if the mobilization technique has not been previously empirically tested (e.g., airplanes with reminder to vote advertisements). If the treatment is an oft-used mobilization technique that is extensively studied in the past (e.g., canvassing), then the planner might center a prior around the estimated effects in previous experiments. Although the formalization of subjective belief as a form of probability distribution is a difficult topic and is beyond the scope of this paper, we note that the influence of prior belief diminishes as the size of experimental data increases.

Once a prior belief is elicited and a randomized field experiment is conducted, these two sources of information are combined via Bayes's rule to obtain the posterior belief about the effects of mobilization strategies on different voters. This calculation is done to estimate heterogeneous treatment effects, and one specific approach is proposed in this paper. When this step is complete, for every voter the planner has an updated belief about the most cost-effective way to mobilize that voter. Finally, the optimal strategy can be derived by optimizing the expected turnout (or the probability of winning in the case of a partisan GOTV planner) after taking into account both overhead and per-voter costs associated with each mobilization technique. Now, we turn to the formalization of our proposed framework.

\footnotetext{
${ }^{1}$ In what follows, we describe our methodology as if a campaign planner both analyzes the data and makes the decision. Clearly, this does not have to be the case since our methods apply directly to situations where a planner makes the decision based on the recommendations given by a data analyst.
} 


\subsection{The Planner's Decision Problem}

We begin our analysis by formally stating the decision problem faced by a nonpartisan GOTV campaign planner. Let $\mathcal{P}$ denote this planner's target population of voters where this population is assumed to be finite and of size $N$. Typically, the target population is the registered voters in the electoral district, whose complete list is available to the planner (at least in the United States). Then the planner's decision problem is to assign one of $K$ available mobilization techniques (i.e., treatments) to each member $i$ of this population. If a GOTV campaign may be planned at the levels of households or precincts, then $i$ indexes appropriate units rather than individual voters. Although we assume that the units of analysis are individual voters for notational simplicity, our proposed methodology is directly applicable to aggregate units.

We use an unordered set $\mathcal{T} \equiv\{0,1, \ldots, K-1\}$, where $K \geqslant 2$, to denote the finite set of mobilization techniques from which the planner makes a selection for each voter in $\mathcal{P}$. Note that $T=0$ represents the strategy of not mobilizing (i.e., doing nothing). For example, the planner may consider three strategies (i.e., $K=3$ ), where $T=2$ and $T=1$ represent a GOTV method based on a phone call and a postcard, respectively, and $T=0$ denotes a status quo strategy that involves no such phone call or mailing. Alternatively, mobilization techniques may differ in their frequency, timing, contents of messages, and other aspects.

Next, suppose that the planner observes the $J$-dimensional (pretreatment) covariates $X$ for each member of this population $\mathcal{P}$. Since $\mathcal{P}$ is a finite population, this means that the planner knows the population distribution of $X$, that is, $P(X)$, whose support is denoted by $\mathcal{X}$. For example, if the target list of voters is obtained from the voter roll, such covariates may include age, gender, voting history, party registration, and the zip code. Since the values of these covariates are observable for every voter on the list, the distribution of $X$ is known to the planner.

Finally, following the statistical literature on causal inference, let $Y_{i}(t)$ represent the potential turnout of voter $i$ that would be realized if the planner applies the mobilization technique $t$ to this voter where $i \in \mathcal{P}$ and $t \in \mathcal{T}$. Then the observed turnout is given by $Y_{i}=Y_{i}\left(T_{i}\right)$. The outcome variable is binary and is equal to 1 if voter $i$ turns out and is equal to 0 otherwise. For the sake of simplicity, we assume no interference between voters (Cox 1958; Rubin 1990). But this assumption can be easily relaxed. If a voter's turnout decision depends on the treatment status of the other voters within the same household (Nickerson 2008), for example, then an analysis needs to be conducted at the household level.

Given this setup, the planner's mobilization strategy is characterized by the following function:

$$
\delta(\cdot, \cdot):(\mathcal{T} \times \mathcal{X}) \mapsto[0,1],
$$

where the mobilization strategy $\delta(t, x)$ denotes the probability of receiving treatment $t \in \mathcal{T}$ for a voter with $X=x$. Alternatively, $\delta(t, x)$ may represent the fraction of voters with the observed covariate $X=x$ who are contacted by the planner using the mobilization method $t$. These two definitions become essentially identical when the number of voters is large but have different implications for the way the optimization is conducted. In either case, voters with the same value of $X$ are assumed to be exchangeable since the planner does not possess additional information to distinguish them. Thus, the planner is interested only in determining the value of the function $\delta(t, x)$ for each value of $t \in \mathcal{T}$ and $x \in \mathcal{X}$. Given this definition, the set of feasible mobilization strategies, $\Delta$, is the collection of functions $\delta(\cdot, \cdot)$ that satisfy the following complement property constraint:

$$
\Delta \equiv\left\{\delta(\cdot, \cdot): \sum_{t=0}^{K-1} \delta(t, x)=1 \text { for every } x \in \chi\right\}
$$

Now, the goal of a nonpartisan GOTV campaign planner is to derive the optimal mobilization strategy so that the turnout of the target population is maximized. The planner may achieve this by deriving the strategy that maximizes the expected turnout given the observed covariate information about $X$. Then the planner's objective function can be written as a function of the planner's mobilization strategy as well as the probability of a voter's turnout given the values of covariates and the actual mobilization strategy applied to the voter, 


$$
g(\delta, \rho) \equiv \mathbb{E}\left(\sum_{i=1}^{N} \delta\left(t, X_{i}\right) Y_{i}(t) \mid X\right)=N \sum_{x \in \mathcal{X}} P(X=x) \sum_{t=0}^{K-1} \delta(t, x) \rho(t, x),
$$

where the turnout profile is denoted by $\rho(\cdot, \cdot):(\mathcal{T} \times \mathcal{X}) \mapsto[0,1]$ with $\rho(t, x) \equiv \operatorname{Pr}(Y(t)=1 \mid X=x)$. The turnout profile represents the turnout probability given the characteristics of a voter and the mobilization strategy applied to this voter. Note that the function $\rho(\cdot, \cdot)$ is unknown to the planner. If the turnout profile were known (and there were no budget constraint), then the planner would apply the mobilization technique $t$ that gives the highest value of the function $\rho(t, x)$ given each voter's covariate characteristics $x$. However, since $\rho(\cdot, \cdot)$ is unknown in practice, the planner must make the decision under uncertainty by learning about $\rho(\cdot, \cdot)$ from the experimental data.

A typical voter mobilization method usually has a small effect on an individual's turnout probability relative to the individual's baseline predisposition to vote. For this reason, planners may cast the objective function of equation (3) in terms of treatment effect rather than turnout profile. In this framework, the planner maximizes

$$
g(\delta, \rho)=\mathbb{E}\left(\sum_{i=1}^{N} \delta\left(t, X_{i}\right)\left(\tau_{i}(t)+Y_{i}(0)\right) \mid X\right) \propto N \sum_{x \in \mathcal{X}} P(X=x) \sum_{t=1}^{K-1} \delta(t, x) \tau(t, x),
$$

where $\tau_{i}(t) \equiv Y_{i}(t)-Y_{i}(0)$ is the treatment effect of mobilization strategy $t$ on voter $i$ and $\tau(\cdot, \cdot):(\mathcal{T}, \mathcal{X}) \mapsto[-1,1]$ with $\tau(t, x) \equiv \operatorname{Pr}(\mathrm{Y}(1)=1 \mid X=x)-\operatorname{Pr}(Y(0)=1 \mid X=x)$. The summation in this equation starts with $t=1$ instead of $t=0$ because $\tau_{i}(0)=0$. This setup is mathematically equivalent to equation (3) in that the optimal GOTV strategy will maximize both objective functions. The decision whether to maximize turnout profile (equation (3)) or treatment effect (equation (4)) hinges on whether the planner can more easily elicit a prior for the turnout profile or treatment effects (see Section 3.1).

Furthermore, in practice, the planner cannot maximize the expected turnout without considering the differing costs of various GOTV techniques. We operationalize this idea by assuming that the planner faces the following budget constraint:

$$
\sum_{t=1}^{K-1} 1\left\{\sum_{x \in \mathcal{X}} \delta(t, x) \neq 0\right\} \kappa(t)+N \sum_{x \in \mathcal{X}} P(X=x) \sum_{t=1}^{K-1} \delta(t, x) \xi(t, x) \leqslant C,
$$

where $1\{\cdot\}$ is the indicator function and $C$ is the fixed positive constant representing the maximum cost allowed for the GOTV campaign. In this formulation, there are two kinds of costs the planner needs to consider. The first is the fixed overhead cost denoted by the function $\kappa(\cdot): \mathcal{T} \mapsto[0, \infty)$ for each mobilization technique $t$. This cost is incurred so long as at least one voter is assigned to the mobilization method. The second component is the cost per voter, that is, the cost of contacting each voter with covariate value $x \in \mathcal{X}$, which is represented by a known cost function $\xi(\cdot, \cdot):(\mathcal{T} \times \mathcal{X}) \mapsto[0, \infty)$. Thus, the planner must determine which mobilization technique is most cost effective for different voters and whether such differences are large enough to warrant use of multiple mobilization techniques. Finally, we note that since $t=0$ corresponds to the status quo strategy of not mobilizing, both the overhead and pervoter costs are zero for this strategy, that is, $\xi(0, x)=\kappa(0)=0$ for all $x \in \mathcal{X}$. This is why the summation in equation (5) is taken with all possible values of $t$ except $t=0$. For the other mobilization strategies, we assume that the cost per voter is positive, that is, $\xi(t, x)>0$ for all $x \in \mathcal{X}$ and $t>0$, whereas the overhead cost is nonnegative, that is, $\kappa(t) \geqslant 0$ for all $t>0$.

\subsection{Data from a Randomized Field Experiment}

Given the above decision theoretic framework, the planner can analyze the data from a randomized field experiment to derive the optimal GOTV campaign. Here, we formalize the assumptions required to link a field experiment with a planner's decision problem. First, we assume that the experiment is conducted on a representative sample of size $n$ taken from the $\operatorname{target}$ population $\mathcal{P}$. Without such an assumption, the planner must model the nonrandom sample selection mechanism in order to infer $\mathcal{P}$ from the experiment. We 
observe the data denoted by $D=\left\{\tilde{Y}_{i}, \tilde{T}_{i}, \tilde{X}_{i}\right\}_{i=1}^{n}$, where $\tilde{Y}_{i}$ is the binary turnout variable, $\tilde{T}_{i} \in \mathcal{T}$ is the treatment variable representing mobilization techniques, and $\tilde{X}_{i} \in \mathcal{X}$ is the same set of covariates as before. The potential outcomes are denoted by $\tilde{Y}_{i}(t)$, where $\tilde{Y}_{i}=\tilde{Y}_{i}\left(\tilde{T}_{i}\right)$ for $t \in \mathcal{T}$.

In practice, this assumption of representativeness may limit the use of a field experiment for deriving an optimal policy. There are two basic ways to address this problem. The first approach is to change the target population $\mathcal{P}$ in such a way that the assumption holds. For example, if a field experiment is conducted only on a random sample of registered voters, then a planner may apply the optimal policy to the population of registered voters alone and exclude nonregistered voters. Another possibility is the use of extrapolation based on the other available covariates. For example, a planner may assume that treatment effect heterogeneity identified among registered voters can be applied to nonregistered voters so long as their age, gender, and education levels are the same. More research is needed for identifying the situations when such an assumption can be made (see Cole and Stuart 2010; Hartman, Grieve, and Sekhon 2010; Green and Kern 2010a).

Another key assumption required for the planner to apply the results of a randomized field experiment to the decision problem is that the joint distribution of potential outcomes and covariates does not change, that is, $P(\tilde{Y}(t), \tilde{X})=P(Y(t), X)$. The assumption may be violated if, for example, the election in which the experiment was conducted differs significantly from the election for which the planner is designing the GOTV campaign. Although in real-world settings this assumption may hold only approximately, it is a necessary requirement for learning about the planner's decision problem from a field experiment.

If the above assumptions hold, the randomization of treatments in field experiments imply that the turnout profile, $\rho(\cdot, \cdot)$, is identified, that is, $\rho(t, x)=P(\tilde{Y}(t)=1 \mid \tilde{X}=x)$, for all $t \in \mathcal{T}$ and $x \in \mathcal{X}$. Although large-sample identification results such as this one are important, in practice the planner must estimate $\rho(\cdot, \cdot)$ from a finite sample and make the decision under uncertainty. (Similarly, we can identify $\tau(t, x)$ but must estimate it from a finite sample.) Next, we address this problem by showing how to derive the optimal nonpartisan GOTV campaign strategy in this setting when the planner is assumed to be Bayesian.

\subsection{The Bayesian Planner}

We assume that the planner is Bayesian and has a prior belief on the space of functions of $\rho(\cdot, \cdot)$. This prior distribution is denoted by $\pi(\rho)$. The Bayesian planner will update her belief via Bayes's rule after observing the data from the randomized field experiment described above. We use $\pi(\rho \mid D)$ to represent this posterior belief about the turnout profile. In the Bayesian statistical decision framework (e.g., Berger 1985), the optimal nonpartisan GOTV strategy $\delta^{*}$ maximizes the posterior mean of the expected turnout, that is,

$$
\delta^{*}=\underset{\delta \in \Delta}{\operatorname{argmax}} \int g(\delta, \rho) \mathrm{d} \pi(\rho \mid D),
$$

where the optimization is subject to the budget constraint given in equation (5).

There are several reasons why we use the Bayesian optimality criteria rather than a frequentist approach based on maximin or minimax regret criteria that are popular in the treatment choice literature. First, at the most intuitive level, the Bayesian criteria allows for the direct incorporation of prior information that academic researchers and practitioners may possess. This is especially appealing in the current context because experienced GOTV campaign planners may actually possess such information and academic researchers may also obtain it from the accumulation of empirical evidence from existing field experiments.

Second, at a more technical level, the Bayesian decision has a frequentist justification. To see this, consider an alternative class of GOTV mobilization strategies that directly depend on the experimental data as well as the values of observed covariates. Such strategies are called "statistical treatment rules" and are characterized by the function $\delta(\cdot, \cdot, \cdot):(\mathcal{T} \times \mathcal{X} \times \mathcal{D}) \mapsto[0,1]$ (Manski 2005). Thus, the set of feasible strategies $\Delta$ equals the set of all functions $\delta(\cdot, \cdot, \cdot)$ that satisfy $\sum_{t=0}^{K-1} \delta(t, x, D)=1$ for all $(x, D) \in(\mathcal{X} \times \mathcal{D})$. Under this setting, the frequentist objective function (i.e., risk) is given by 


$$
g\left(\delta, F_{D}, \rho\right)=\int_{D} N \sum_{x \in \mathcal{X}} P(X=x) \sum_{t=0}^{K-1} \delta(t, x, D) \rho(t, x) \mathrm{d} F_{D}
$$

with the following budget constraint:

$$
\sum_{t=1}^{K-1} 1\left\{\sum_{x \in \mathcal{X}} \delta(t, x, D) \neq 0\right\} \kappa(t)+N \sum_{x \in \mathcal{X}} P(X=x) \sum_{t=1}^{K-1} \delta(t, x, D) \xi(t, x) \leqslant C,
$$

for all $D \in \mathcal{D}$. Again, the costs for the status quo strategy $t=0$ are zero and excluded from the budget constraint. It has been shown that in most practically relevant situations, the Bayesian decision $\delta^{*}$ defined in equation (6) agrees with the decision that maximizes the expected value of $g\left(\delta, F_{D}, \rho\right)$ averaging over the prior distribution of $D$ on $\mathcal{D}$ (see Berger 1985, 159; Manski 2005, 59). Also, if the size of experimental data is large (as in many GOTV randomized field experiments) and little prior information is available, the Bayesian decision is essentially equivalent to the strategy that maximizes the expected turnout. Thus, the Bayesian decision can be justified from a frequentist perspective.

Third, an alternative optimality criterion that attracted attention in the literature is the minimax regret principle of Wald (1950) (see Savage 1951; Manski 2005). One important advantage of the minimax regret criterion is that it avoids the subjectivity of Bayesian optimality because it does not require the use of prior information. On the other hand, unlike the Bayesian decision theory, the frequentist theory based on the minimax regret criterion typically does not lead to the unique optimal decision, which practitioners may find problematic. In addition, the strategies that meet the minimax regret criterion can include no-data rules that do not depend on the data at all (Stoye 2009). Clearly, such strategies are not desirable as they do not allow the planner to learn anything from the available experimental data. Furthermore, a minimax regret rule can be viewed as a Bayes rule with a prior (i.e., a least favorable prior) distribution (Berger 1985, chap. 5). Thus, depending on the planner's subjective belief, the Bayesian GOTV strategy can meet the minimax regret criterion.

\section{The Optimal Nonpartisan Campaign Strategy}

Given the decision theoretic framework described in the previous section, we next show how to derive the optimal GOTV campaign strategy defined in equation (6). One relative advantage of the proposed Bayesian framework over a frequentist's approach is that we can completely separate the derivation of the optimal campaign strategy from the analysis of the experimental data. That is, we first use statistical models to obtain the posterior belief of the turnout profile, $\rho(\cdot, \cdot)$. Conditional on this posterior turnout profile, we obtain the optimal campaign strategy by solving an optimization problem subject to a budget constraint.

\subsection{The Statistical Method for Estimating Heterogenous Treatment Effects}

There are many statistical models that could be used to obtain the posterior distributions of the turnout profile. Nevertheless, when choosing a model, the following statistical issues need to be addressed. First, a statistical model needs to be flexible in order to avoid strong functional form assumptions. In particular, a nonparametric model is useful when a planner does not know the exact functional form of the turnout profile a priori. Second, a statistical model should be able to accommodate discrete covariates, which are often the only ones available to planners, such as in GOTV field experiments (e.g., party registration, previous turnout history). This excludes the use of certain binary nonparametric regression models that require covariates to be continuous. Third, the model fitting procedure should only require a minimal number of arbitrary decisions from a planner or a data analyst who is working for the planner.

Finally, a model should not be overfitted to the data at hand and thus an appropriate set of pretreatment covariates must be carefully selected. Overfitting tends to yield a model with poor performance in the actual election to which the derived mobilization strategy will be applied. This is the main problem of the post hoc subgroup analysis as emphasized in Section 1. In practice, this consideration is important because the sample size may not be large enough to accommodate a large number of pretreatment 
covariates that are potentially useful for deriving the optimal campaign strategy. Thus, an appropriate variable selection algorithm is needed as a part of the model selection procedure.

Moreover, as Gunter, Zhu, and Murphy (2007) point out, most existing variable selection algorithms are developed for improving prediction rather than decision making. They are closely related but not the same concepts. For example, a powerful predictor of the outcome, that is, a predictive variable, is not necessarily useful for decision making if its effect on the outcome is constant between the treatment and control groups. Similarly, a variable that explains a significant portion of treatment effect heterogeneity, that is, a prescriptive variable, may not be selected by standard variable selection procedures if it does not predict the observable outcome (rather than the potential outcome) as well as other variables.

Below, we describe a method that meets the above criteria relatively well. Our method is similar to the one proposed by Gunter, Zhu, and Murphy (2007) but differs from it in that we use a tree-based method (Breiman et al. 1984) rather than Lasso, and our measure of importance for prescriptive variables is somewhat different. ${ }^{2}$

One advantage of tree-based methods in the context of GOTV studies is that the methods divide the sample into various subgroups, making it easier for practitioners to interpret the results. Also, tree-based methods can handle categorical variables better than other methods, and this fits most GOTV studies where many covariates are categorical.

To introduce our proposed method, we begin by describing a simple Bayesian approach to model turnout among voters with the same characteristics of covariates via the binomial distribution. For the moment, we assume that the sample size is sufficiently large and thus there is no need for variable selection. Using a conjugate prior, we can write the model as

$$
\begin{gathered}
W_{t x} \mid T=t, X=x \sim \operatorname{Binom}\left(n_{t x}, \rho(t, x)\right), \\
\rho(t, x) \mid X=x \sim \operatorname{Beta}\left(a_{t x}, b_{t x}\right),
\end{gathered}
$$

for each $t \in \mathcal{T}$ and $x \in \mathcal{X}$, where $W_{t x}$ is the number of voters with $T_{i}=t$ and $X_{i}=x$ who turned out, $n_{t x}$ is the total number of such voters, and $\left(a_{t x}, b_{t x}\right)$ are the prior parameters. It is well known that this model yields the following posterior distribution:

$$
\rho(t, x) \mid Y_{i}, X_{i}=x \sim \operatorname{Beta}\left(W_{t x}+a_{t x}, n_{t x}-W_{t x}+b_{t x}\right),
$$

where the posterior mean of $\rho(t, x)$ is given by $\left(W_{t x}+a_{t x}\right) /\left(n_{t x}+a_{t x}+b_{t x}\right)^{3}$

Although its simplicity is attractive, the above model is unlikely to work well in practice if the sample size is small relative to the number of unique values the observable covariates $X$ take. In particular, if one conditions upon irrelevant covariates, then the sample size within each subgroup will be too small to yield informative inferences about $\rho(t, x)$. Such overfitting will then necessarily lead to a mobilization strategy whose performance in the actual election will be poor. On the other hand, if important covariates are not used to define subgroups, the planner will fail to differentiate across voters and will choose a suboptimal campaign strategy. Thus, as explained earlier, in most practical cases we seek a principled way to select relevant variables and form subgroups before applying the above standard Bayesian model.

Our proposed solution to this problem consists of the following three steps. First, a variable selection algorithm is applied in order to decide which variable needs to be conditioned upon when deriving the optimal campaign strategy. Next, given the selected variables, a tree-based classification method (Breiman et al. 1984) is fitted to each treatment/control group in order to identify relevant subgroups within the group. ${ }^{4}$ We use a cross-validation procedure to avoid overfitting. Finally, once all subgroups are identified in this way, we then apply the above Binomial-Beta model within each subgroup to obtain the posterior distribution of $\rho(t, x)$ for all $t$ and $x$. We call this approach semi-Bayesian because the data are used

\footnotetext{
${ }^{2}$ It is also possible to use a Bayesian version of regression tree models (Chipman, George, and McCulloch, 2010; Green and Kern, 2010b; Hill forthcoming) and of course many other data mining methods.

${ }^{3}$ Alternatively, the planner may formalize a prior belief in terms of treatment effects. If a normal prior distribution is used, then the posterior of $\tau(t, x)$ can also be approximated by a normal distribution.

${ }^{4}$ This tree-based classification method is one of many nonparametric models one can use. One disadvantage of this approach is that gradual changes in treatment effects across covariate groups are modeled as sharp discontinuities rather than smooth functionals.
} 
twice - to form subgroups and then calculate the posterior distribution. Below, we present the details of our proposed method.

Step 1: (Selection of Predictive Variables) Fit a classification tree to the entire sample using all pretreatment covariates and the treatment variable. Use $K$-fold cross validation on the misclassification rate to determine the value of the parameter which controls the complexity of the tree, for example, the complexity parameter in rpart() implementation in R (Ripley 1996, chap. 7). Denote the predictive variables that are used in the final model by $V$, that is, $V \subset X$.

Step 2: (Importance of Prescriptive Variables) Order each pretreatment covariate $X_{j}$ for $j=1,2, \ldots, J$, based on the statistic $S_{j} \equiv g_{j 1}^{*}-g_{j 0}^{*}$, where $g_{j k}^{*}$ is the optimal overall turnout using the turnout profile $\hat{\rho}_{j k}(t, x)$ for $k=0,1$. We obtain $\hat{\rho}_{j 1}(t, x)$ by fitting a classification tree for the treated subset of the data (i.e., $\tilde{T}_{i} \geqslant 1$ ) and using $V$ and $X_{j}$ as covariates. Similarly, $\hat{\rho}_{j 0}(t, x)$ is obtained by fitting a classification tree on the untreated subset of the date (i.e., $\tilde{T}_{i}=0$ ). Note that when fitting these trees, we use the value of the complexity parameter from Step 1.

Step 3: (Model Fitting) For each $j=1, \ldots, J$ with $S_{j}>0$, conduct the following:

(a) Randomly divide the sample into $K$ subsets for $K$-fold cross validation.

(b) Using $K-1$ training sets, fit classification trees (without pruning) separately to the treatment and control groups using $V$ and $j$ most important prescriptive variables in both models. Select the values of the complexity parameters for the two models based on the mean of the optimal overall turnout across $K$ validation sets. Denote the optimal overall turnout and the optimal campaign strategy corresponding to the selected values of complexity parameters by $g_{j}^{*}$ and $\delta_{j}^{*}$, respectively.

Step 4: (Derivation of Optimal Strategy) The optimal overall turnout is given by $g^{*}=\max _{1 \leqslant j \leqslant J} g_{j}^{*}$, whereas the optimal campaign strategy is given by $\delta^{*}=\delta_{\operatorname{argmax}_{1 \leqslant j \leqslant \leqslant} g_{j}^{*}}^{*}$

Each step of the above algorithm can be understood as follows. The first step selects predictive variables using a standard fitting procedure of tree-based methods. The second step orders each pretreatment covariate (including the ones identified as predictive variables in Step 1) according to its importance as a prescriptive variable. The statistic, $S_{j}$, is designed to measure how much a planner can increase the optimal overall turnout by interacting the value of $X_{j}$ with treatment. This statistic provides a measure of the ability of $X_{j}$ to explain heterogeneous treatment effects. The third step uses the $K$-fold cross-validation procedure, given all predictive variables and different subsets of prescriptive variables, in order to select the values of complexity parameters for classification trees fitted separately to the treatment and control groups. This is done by comparing the optimal overall turnout corresponding to different values of the complexity parameters. Finally, the fourth step selects the final model among the ones chosen in Step 3 by again comparing the resulting optimal overall turnout and thus determines the optimal campaign strategy.

A main advantage of this semi-Bayesian approach is that it inherits the simplicity of tree-based methods. In particular, each of the subgroups that result from the final model is interpretable by practitioners. This means that it is possible to use available prior information within subgroups by specifying the parameters of the beta prior distribution. The proposed approach also addresses two key issues mentioned at the beginning of this section. The tree-based classification models, which our method uses, are nonparametric and can handle discrete covariates in an effective manner. The use of cross-validation procedure avoids overfitting. Finally, transparent algorithms such as the one proposed here prevent planners from making arbitrary decisions when deriving the optimal campaign strategy.

\subsection{The Derivation of the Optimal Mobilization Strategy}

Given the posterior turnout profile obtained above, we next show how to derive the optimal nonpartisan GOTV campaign strategy. Let $\tilde{\rho}(t, x)$ be the posterior turnout profile for each $t \in \mathcal{T}$ and $x \in \mathcal{X}$. Then the optimal campaign strategy can be obtained by solving the following constrained optimization problem:

$$
\delta^{*}=\underset{\delta \in \Delta}{\operatorname{argmax}} N \sum_{x \in \mathcal{X}} P(X=x) \sum_{t=0}^{K-1} \delta(t, x) \tilde{\rho}(t, x),
$$

subject to the budget constraint given in equation (5). 
To solve this optimization problem, we first consider the case of no budget constraint. In this case, the solution is trivial because one simply needs to assign the most effective strategy to each strata defined by $X$. That is, the optimal campaign strategy is given by

$$
\delta^{*}(t, x)= \begin{cases}1 & \text { if } t=\operatorname{argmax}_{s \in \mathcal{T}} \tilde{\rho}(s, x) \\ 0 & \text { otherwise }\end{cases}
$$

for any given $x \in \mathcal{X}$. However, in many cases, the budget constraint may prevent the planner from employing campaign strategy $\delta^{*}$ defined in equation (13).

Next, consider the case where the budget constraint is binding so that the strategy defined by equation (13) is not feasible and yet there is no overhead cost. Unfortunately, in this case, the derivation of the optimal strategy is no longer trivial. Thus, as a general strategy, we use the fact that this is the following constrained linear optimization problem:

$$
\begin{gathered}
\delta^{*}=\underset{\delta \in \Delta}{\operatorname{argmax}} N \sum_{x \in \mathcal{X}} P(X=x) \sum_{t=0}^{K-1} \delta(t, x) \tilde{\rho}(t, x), \\
\text { subject to }\left\{\begin{array}{l}
\delta(t, x) \geqslant 0 \quad \text { for all } x \in \chi \text { and all } t \in \tau, \\
\sum_{t=0}^{K-1} \delta(t, x)=1 \quad \text { for all } x \in \chi, \\
N \sum_{x \in \chi} P(X=x) \sum_{t=1}^{K-1} \delta(t, x) \xi(t, x) \leqslant C .
\end{array}\right.
\end{gathered}
$$

Then, the standard linear programming algorithm can be used to obtain the optimal strategy, $\delta^{*}$. If $\delta(t, x)$ represents the proportion of voters rather than the probability of treatment assignment, then this can be formulated as a mixed integer programming problem, ${ }^{5}$ which is more difficult but can be solved by applying an appropriate algorithm. Moreover, as the sample size increases, this difference vanishes.

Finally, when there exists overhead costs for the mobilization methods, we consider a case where only a subset of mobilization techniques are applied to at least one voter. In this case, we can solve the optimization problem in the same manner as the case without the overhead cost except that the total overhead cost is subtracted from the maximum budget allowed for the campaign, that is, $C-\sum_{t=1}^{K-1} 1\left\{\sum_{x \in \mathcal{X}} \delta(t, x) \neq 0\right\} \kappa(t)$. The optimal strategy is obtained by considering all possible such subsets and their corresponding optimal strategy and then choosing the strategy that yields the greatest posterior expected turnout.

Although the proposed general strategy based on linear programming is easy to implement, in some cases, an approximate method, which is more computationally efficient, may be preferred. In Appendix A (supplementary material), we consider a fast approximate solution to the nonpartisan planner's optimization problem.

\section{The Optimal Partisan Campaign Strategy}

In this section, we extend the proposed decision theory framework as well as the statistical and optimization methods described above to the case of a partisan persuasion and GOTV campaign planner. ${ }^{6} \mathrm{We}$ show how a Bayesian planner can derive the optimal campaign strategy using randomized field experiments in order to maximize the (posterior) expected chance of winning the election. Throughout the section, we assume that two major candidates compete for the office. Minor party candidates may exist, but it is assumed that their probability of winning the election is negligibly small.

\footnotetext{
$\overline{{ }^{5} \mathrm{~A} \text { linear programming }}$ problem is called a mixed integer programming problem if some unknown parameters are required to be integers.

${ }^{6}$ A GOTV campaign that disproportionately drives out one candidate's vote affects the outcome of the election, hence we reference GOTV campaigns in this section as well.
} 


\subsection{The Decision Problem}

Using the same notation introduced in Section 2, the decision problem of the partisan campaign planner is to assign one of $K$ different mobilization methods (including the status quo strategy of doing nothing, which is denoted by $T_{i}=0$ ) to each member of the target population $\mathcal{P}$ of finite size $N$. Again, we assume that the planner knows the distribution of a certain set of covariates $P(X)$. Thus, the planner's mobilization strategy can be characterized by $\delta(\cdot, \cdot)$ in the same way as before (see equation (1)) and the set of feasible such strategies is equal to $\Delta$ which is defined in equation (2).

Unlike a nonpartisan campaign planner, however, a partisan campaign planner wishes to choose the mobilization strategy that leads to the election victory. For this decision problem, the outcome variable $Y_{i}$ needs to be redefined in the following manner. Let $Y_{i}(t)$ represent the potential voting behavior of voter $i$ that would be realized if the planner assigns mobilization method $t$ to this voter where $i \in \mathcal{P}$ and $t \in \mathcal{T}$. The variable $Y_{i}(t)$ can take three different values; it equals 1 if voter $i$ casts a ballot for the candidate of the planner's party, -1 if she votes for the opponent, and 0 otherwise (voting for a third-party candidate or abstaining, etc.). Then the planner's ultimate goal is to win the election, which can be represented as the following indicator function:

$$
h(\delta, V) \equiv 1\left\{\sum_{i=1}^{n} \delta\left(t, X_{i}\right) Y_{i}(t)>0\right\}=1\left\{\sum_{x \in X} P(X=x) \sum_{t=0}^{K-1} \delta(t, x) v(t, x)>0\right\}
$$

where $v(t, x) \equiv \sum_{i \in\left\{i^{\prime}: X_{i^{\prime}}=x\right\}} Y_{i}(t) / \sum_{i=1}^{N} 1\left\{X_{i}=x\right\}$ is a random variable representing the vote share differential for the candidate that would result among voters with covariates $X=x$ if the planner assigns mobilization method $t$ to them. Clearly, $h(\delta, V)$ is equal to 1 if the candidate of the planner's party wins the election and it is equal to 0 if he/she loses. In the statistical decision theory literature, such an objective function is called " $0-1$ loss function."

Finally, the partisan planner typically faces a budget constraint similar to the one confronted by the nonpartisan planner, and therefore, we use equation (5) for the partisan planner.

\subsection{The Data Requirements}

As is the case for a nonpartisan GOTV campaign planner, the assumptions are required in order for a partisan campaign planner to be able to directly use randomized field experiments for the optimal decision. These assumptions are essentially identical to those described in Section 2.3. That is, we require that (1) a field experiment is conducted on a representative sample from the same target population of voters $\mathcal{P}$ (but see Cole and Stuart 2010; Hartman, Grieve, and Sekhon 2010; Green and Kern 2010a) and (2) the joint distribution of potential outcomes and covariates, $P(Y(t), X)$, remains identical between the experiment and the actual election. However, one important difference is that the derivation of the optimal partisan campaign requires vote choice data as well as turnout data for the voters who are subjects of field experiments; recall that in the case of partisan campaign $Y(t)$ represents a trichotomous variable rather than a binary variable. For example, in the United States, unlike turnout data, vote choice data are not publicly available and cannot be verified for each voter. This means that a sample survey needs to be conducted in order to derive the optimal partisan mobilization strategy unless the entire analysis and strategy planning are conducted at an aggregate level where validated election results are available.

\subsection{The Derivation of the Optimal Strategy}

Using the Bayes theorem, we derive the optimal partisan campaign strategy just as done in the case of nonpartisan GOTV campaign. In particular, the optimal strategy maximizes the posterior probability of winning the election,

$$
\delta^{*}=\underset{\delta \in \Delta}{\operatorname{argmax}} \int h(\delta, v) \mathrm{d} \pi(v \mid D),
$$

subject to the budget constraint given in equation (5), where $\pi(v \mid D)$ is the posterior distribution of the vote share differential $v(\cdot, \cdot)$. Using the classification method and variable selection algorithm similar to those 
described in Section 3.1, we can estimate $v(\cdot, \cdot)$ except that the outcome variable is now trichotomous rather than binary. Most existing classifiers including tree-based methods can handle such categorical variables even when the number of categories is greater than two.

The problem, however, is that the optimization in equation (17) is not trivial for two reasons. First, the integration cannot be explicitly solved. Second, the objective function is an indicator function that is not continuous. These difficulties are amplified by the fact that the optimization must be conducted over a high-dimensional space if the number of treatments and/or the number of subclasses is large. These computational considerations prevented Kramer (1966), who studied this problem more than 40 years ago, from using the probability of winning as the objective function of a partisan campaign planner. Instead, he used the expected plurality of votes as the objective function while acknowledging that it may not be appropriate. Kramer (1966) explained this dilemma as follows: "the probabilistic objective is the more realistic. However, this formulation is computationally quite difficult to work with" (141). Indeed, if the partisan planner wishes to maximize the expected plurality, one can solve a constrained optimization problem by applying the standard linear programming algorithm as explained in Section 3.2. ${ }^{7}$

To overcome this computational difficulty, we use a fast and approximate solution to the partisan planner's optimization problem to maximize the posterior probability of winning. This method, detailed in Appendix B (supplementary material), is not guaranteed to yield an optimal campaign strategy in some situations but is relatively fast and approximates an optimal strategy.

\section{Empirical Evaluation of the Proposed Method}

How effective is the proposed method of deriving the optimal GOTV campaign work in real-world applications? We empirically evaluate the performance of the proposed methods by analyzing three existing data sets of GOTV randomized field experiments. The basic idea here is to use a randomly selected subset of the data as a test data set and obtain an unbiased estimate of the actual turnout (or the probability of winning) by applying the resulting optimal campaign strategy derived from the rest of the data to this test data set. This procedure mimics the real-world situation by using the test data set as the actual election to which the optimal campaign strategy is applied. Since the treatment is randomized and the test data set is not used to derive the optimal strategy, the procedure results in an unbiased evaluation of empirical performance of the proposed methodology.

Our three applications vary in their scope and context in order to demonstrate the wide applicability of our method. The first application is a single-treatment, text messaging nonpartisan GOTV experiment from the 2006 Congressional election. With only one treatment, the decision for the planner is which voters to treat with text messaging. Our second application is a multi-treatment, nonpartisan GOTV effort that used mailings to pressure citizens to vote in the August 2006 primary election in Michigan. Here, the planner must decide which voter to contact and which mobilization method to use when contacting each voter. Finally, the third application is a partisan study with a single mobilization method where the planner's goal is to maximize the probability of winning the election.

\subsection{Evaluation Method}

To assess the effectiveness of the proposed method, we add an additional level of cross validation to the procedure of Section 3. The key idea is to cross validate the whole procedure consisting of the three steps

\footnotetext{
${ }^{7}$ Specifically, the optimization problem is given by

$$
\delta^{*}=\underset{\delta \in \Delta}{\operatorname{argmax}} N \sum_{x \in \mathcal{X}} P(X=x) \sum_{t=0}^{K-1} \delta(t, x) \mathbb{E}(v(t, x) \mid D),
$$$$
\text { subject to } \begin{cases}\delta(t, x) \geqslant 0 & \text { for all } x \in \chi \text { and all } t \in \tau, \\ \sum_{t=0}^{K-1} \delta(t, x)=1 & \text { for all } x \in \chi, \\ N \sum_{x \in \chi} P(X=x) \sum_{t=1}^{K-1} \delta(t, x) \xi(t, x) \leqslant C .\end{cases}
$$ 
described in Section 3.1 and obtain an unbiased estimate of the resulting turnout under the optimal strategy from test data that are not used for the derivation of the strategy. That is, after randomly dividing the sample into $L$ subsamples, we set aside one of them as a test set and apply our methodology to the rest of the data. The derived optimal strategy is then applied to the test set in order to obtain an unbiased estimate of the resulting overall turnout under this strategy. The random assignment of treatments and the random subsampling of the test set make unbiased estimation possible. The entire procedure is repeated $L$ times using each subset as a test set. Finally, the average value of $L$ estimated optimal turnout rates is taken as an estimate of the turnout that would result under our proposed methodology.

Throughout these applications, we use a normal-normal conjugate prior for the treatment effect $\tau$ based on the setup defined in equation (4). The prior for each subgroup treatment effect distribution is Gaussian and centered on the estimated population ATE. The value of the prior variance is chosen so that it increases in proportion to the per capita budget constraint. In each case, a grid search is implemented to approximate the optimal complexity parameter. A 10-fold cross-validation procedure is used for Step 3 of Section 3.1 to determine the optimality of each complexity parameter. In addition, the optimization problem is solved using the algorithms detailed in Appendix (supplementary material) which yield approximate (but fast) solutions.

\subsection{A Nonpartisan GOTV Campaign with a Single Mobilization Method}

During the 2006 election, two nonpartisan organizations contributed the cell phone numbers of newly registered individuals to an experiment that tested the efficacy of text messages to mobilize voters (see Dale and Strauss 2009, for details). The election was of moderate interest, with at least one gubernatorial or senatorial campaign on the ballot in most states. Subjects were included in the experiment when they registered to vote with a campus representative of the Student Public Interest Research Groups (PIRGs) or when they registered online with Working Assets. About 8000 subjects nationwide were randomly assigned, with 50\% probability, to either the treatment or the control group.

The treatment group received a short text message the day before Election Day. An example text message reads "A friendly reminder that TOMORROW is Election Day. Democracy depends on citizens like you-so please vote!-PIRG." The text message appeals were varied slightly, but these differences are ignored for this analysis. Subjects were matched to the voter file using information on their registration forms. The outcome variable is dichotomous: 1 for having voted in 2006 and 0 for not voting. The estimated ATE, or more precisely the overall intent-to-treat effect, was 3.0 percentage points with a standard error of 1.1. Available covariates for our analysis include gender, age, race, past voting history, logged county population density, and registering organization.

We follow the procedure outlined in Section 3 and derive the optimal campaign strategy using this experimental data set. The classification tree produced in Step 1 chose the following variables as having predictive power (i.e., v): age, log of county density, registering organization, whether the subject had voted in a previous election, and gender. The prescriptive variables chosen in Step 2 are age, density, and Hispanic (in decreasing order of $S_{j}$ ). Not all these variables are included in the final classification trees produced since these trees are based on subsets of the data; this smaller sample size reduces the complexity of these final trees. ${ }^{8}$

The resulting final trees are presented in Fig. 2. The tree for the control group is on the left; the tree for the treatment group is on the right. Left branches represent voters who meet the criteria of the nodes; right branches represent voters who would falsify the nodes' inequalities. The leaves show the predicted probability of voting conditional on the leaves' covariates; higher probabilities are to the right at each node. The control group tree demonstrates that, in this group, voters above the age of 24 are predicted to vote at a rate of $62 \%$, while whether the participant's age is known and county population density are important determinants of the voting rates of voters aged 24 years or younger. In the treatment group, the age cutpoint is in between 19 and 20 years, with population density and voting history also providing information about turnout rates.

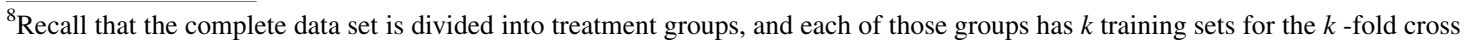
validation. 

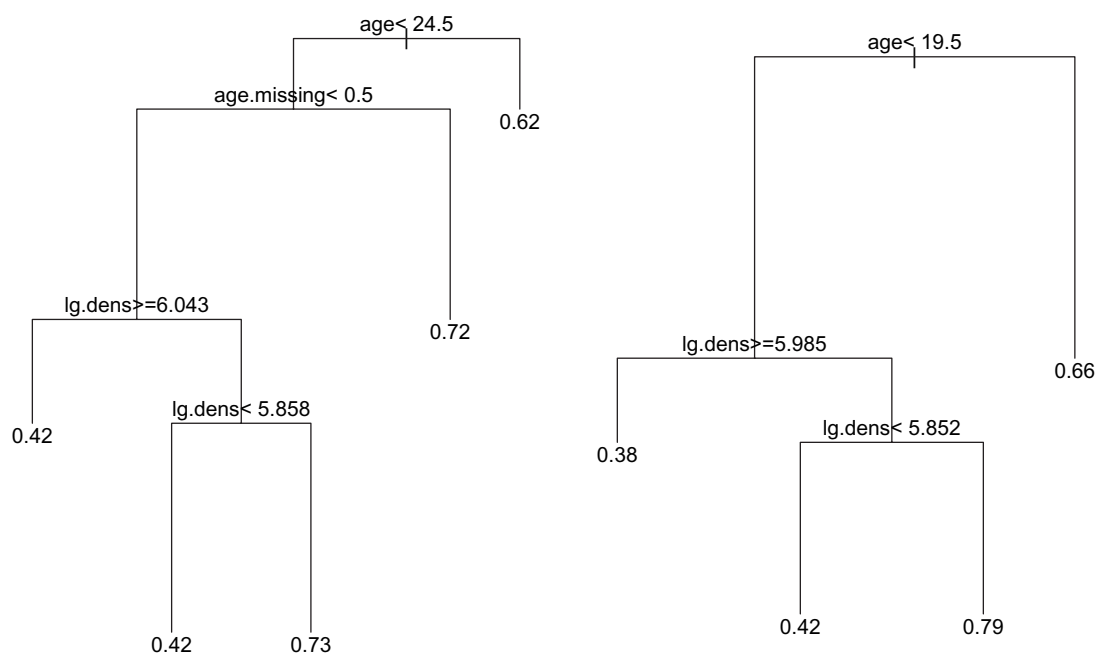

Fig. 2 Final classification trees for the control group (left panel) and treatment group (right panel). The complexity parameters are chosen from 10-fold cross validation using the algorithm described in Section 3.1 so that the resulting optimal turnout is maximized on the validation set. In this example, the planner budget allows treatment of at most $10 \%$ of the population. At each node, subjects who meet the node's criterion are filtered through the left branch of the tree. The leaves show the predicted probability of voting conditional on the leaves' covariates. Covariate abbreviations: age is the age in years of the subject, age.missing is whether the age of the participant is unknown, and lg.dens is the log of the subject's county population density.

Much of the heterogeneous treatment effect can be explained by the voters' ages. Potential voters between the ages of 20 and 24 are very responsive to the treatment. In the control group (left panel of Fig.2), these individuals vote at a rate of $62 \%$. In contrast, the classification tree for the treatment group gives them a predicted probability of voting of $66 \%$ (the right branch of the right panel of Fig. 2) -a 4 percentage-point increase, larger than the ATE of 3 percentage points. Also, the treatment model predicts

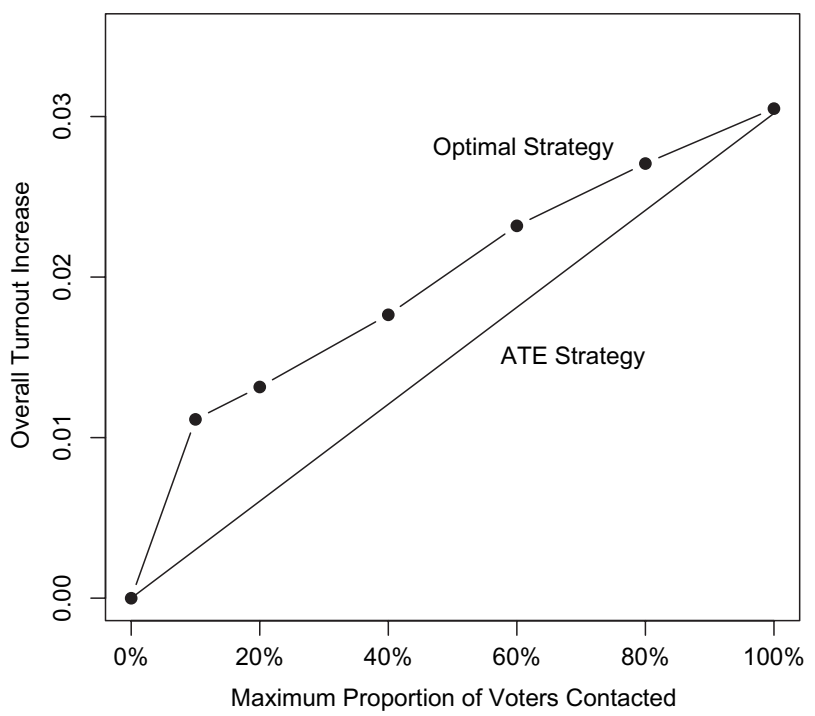

Fig. 3 Empirical evaluation of the performance of the proposed method for the text messaging experiment. The figure displays the overall turnout increase that results from two campaign strategies as a function of the maximum proportion of voters contacted. The first strategy is the ATE strategy (solid line), which contacts randomly selected voters. The second strategy is an optimal approach based on the methodology outlined in this paper, which uses covariate characteristics of voters to determine which voters receive the treatment. Solid circles represent the estimated optimal turnout using the difference-in-means estimator. The estimator is applied to test data that are not used for the derivation of the optimal strategy. 
a negligible or negative treatment effect for 18- and 19-year-olds. This can be seen from the fact that the probability of voting for a majority of this group is predicted to be at most $42 \%$ under the treatment. Note that age ranges such as these could not be identified by a classic model such as logit with linear explanatory variables.

Figure 3 displays the performance of our proposed methodology given the maximum proportion of voters that could be contacted. The turnout that would result under our optimal strategy is estimated using the difference in means estimator between the treated and untreated voters (solid lines with solid circles). That is, we compute the average turnout among the treated voters who are assigned to the treatment group under the optimal strategy as well as the average turnout among the untreated voters who are assigned to the control group under the optimal strategy. The latter is then subtracted from the former to yield the estimated overall turnout under the optimal strategy. The turnout rate achieved with the proposed method compares favorably with the turnout based on a naive strategy where randomly selected voters are contacted. This less-informed strategy, which is solely based on the estimated overall ATE ("ATE strategy"), completely ignores covariate information and thus assumes zero treatment effect heterogeneity.

Figure 3 shows that the application of our proposed method allows one to achieve a higher overall turnout than an ATE strategy (at least on average), if an organization can only afford to treat a subset of the population. For a campaign that can afford to treat $10 \%$ of the population, the optimal campaign strategy achieves an overall turnout on average over three times greater than the turnout increase under the ATE strategy. Because the nonparametric procedure did not isolate individuals who reacted negatively toward the treatment, no gain over the ATE strategy is present when treating the entire population. This lack of negatively responsive individuals is unsurprising given that little backlash was found to the text messages in a posttreatment survey (Dale and Strauss 2008). Overall, for the text messaging application, organizations that desire to pinch pennies would be wise to use the proposed nonparametric procedure, whereas large-budget mobilization campaigns achieve less gain.

\subsection{A Nonpartisan GOTV Campaign with Multiple Mobilization Methods}

In the 2006 Michigan August primary, scholars used the public voting records to test whether social pressure of seeing your or your neighbors' vote records would encourage individuals to vote (Gerber, Green, and Larimer 2008). The effort was extremely successful: learning about your neighbors' vote history increased the probability of casting a ballot from $30 \%$ to $38 \%$. This 8 percentage-point effect of the black-and-white postcard treatment is an order of magnitude above the usual turnout effect of mailings.

Over 300,000 participants are involved in this experiment that had three treatments. The treatments can be ordered in a hierarchy when each successive treatment includes all the aspects of the prior treatments. The civic duty message is common to all treatments. The "Hawthorne treatment" lets individuals know they are being studied. The "Self treatment" implicitly acknowledges that the participant is being studied when it provides the participant's voting record. The "Neighbors treatment" provided information on both the participant's records and those of their neighbors and found to be associated with the largest estimated ATE.

The outcome variable is whether or not the participant voted in the 2006 primary election. Available covariates are age, gender, household size, and voting history (primary election turnout for 2000, 2002, and 2004 and general election turnout for 2000 and 2002 - all participants voted in the November 2004 election by design). The treatment costs are considered equal (all were printed on black-and-white postcards and sent through the U.S. postal service). Thus, the Neighbors condition is the most efficient in terms of votes per dollar. We perform the procedure described in Section 3.2 with the exception that because of the large sample size, 10-fold cross validation is not conducted; instead, a training set of 20,000 participants is applied to a (randomly selected) test set of 125,000 participants.

As shown in Fig. 4, the data exhibit little heterogeneity of treatment response, at least for the given covariates. The dotted line represents the boost in turnout under the non-experimental strategy, that is, the outcome one might expect if the planner does not run an experiment and attempts to mobilize randomly selected voters by randomly selected strategies. The solid line is the turnout boost that would result from treating individuals randomly using the mobilization strategy that yields the best estimated ATE, that is, 


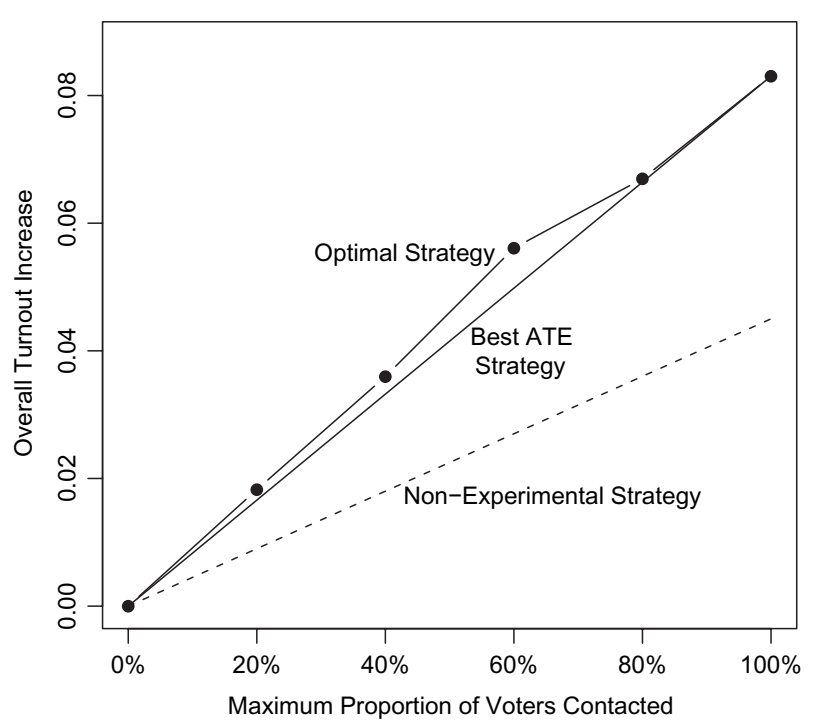

Fig. 4 Empirical evaluation of the performance of the proposed method for social pressure experiment. The figure displays the estimated overall turnout increase that results from three campaign strategies as a function of the maximum proportion of voters contacted. The first strategy (dashed line) is the non-experimental approach in which a mobilization method is chosen randomly and is applied to randomly selected voters ("Non-experimental Strategy"). The second strategy is the non-covariate experimental approach (solid line), which contacts randomly selected voters with the treatment that has the highest average effect ("Best ATE Strategy"). The third strategy is an optimal approach based on the methodology outlined in this paper, which uses covariate characteristics of voters to determine which voters receive the treatment. Solid circles represent the estimated optimal turnout using the difference-in-means estimator. The estimator is applied to (randomly selected) test data $(n=125,000)$ that are not used for the derivation of the optimal strategy (training set $n=20,000$ ).

the outcome expected if the planner conducts an experiment but ignores covariates. The connected circles represent increase in turnout using the information in the covariates. In this example, we find that the covariates are not very helpful, though they do no harm. ${ }^{9}$ For each budget constraint we consider, all participants are optimally treated with the Neighbors treatment. This example demonstrates that using this method even when there is little heterogeneity only helps. Thus, even when a planner is unsure about the level of heterogeneity of the effect of the desired treatment, this procedure can be recommended.

\subsection{A Partisan Persuasion Campaign}

In September 2006, a "well-known liberal activist group" endorsed two Democratic candidates for the Pennsylvania State House and canvassed voters who they thought might be persuadable (see Arceneaux and Kolodny 2009, for details). To assess the effectiveness of their message, randomly selected voters were canvassed. A postelection survey of 2000 respondents recorded the vote preferences of the populations, of which about $30 \%$ received the personal door-to-door canvassing. Unfortunately, the original analysis of the experiment showed that the message miserably failed to persuade voters to support the Democrats. In fact, it ended up mobilizing Republican voters; the average (intention-to-treat) effect was estimated to be -6 percentage points (difference in vote margins) with the standard error of 4 percentage points. Arceneaux and Kolodny (2009) conjecture that the name of the liberal group in the endorsement provided useful information to Republican voters: since the disliked liberal group supported the Democratic candidate, the Republican voters increased their support for the Republican candidate.

To make the evaluation of our proposed method more efficient and realistic, we transform the data in two ways. First, we combine the two districts into one large district (i.e., voting for either Democrat counts as a vote for the Democrat). Second, since the treatment yielded a large and negative effect, we derive the

\footnotetext{
${ }^{9}$ The covariates in the final trees are vote history in the 2002 primary election, 2002 general election, and 2004 primary election.
} 

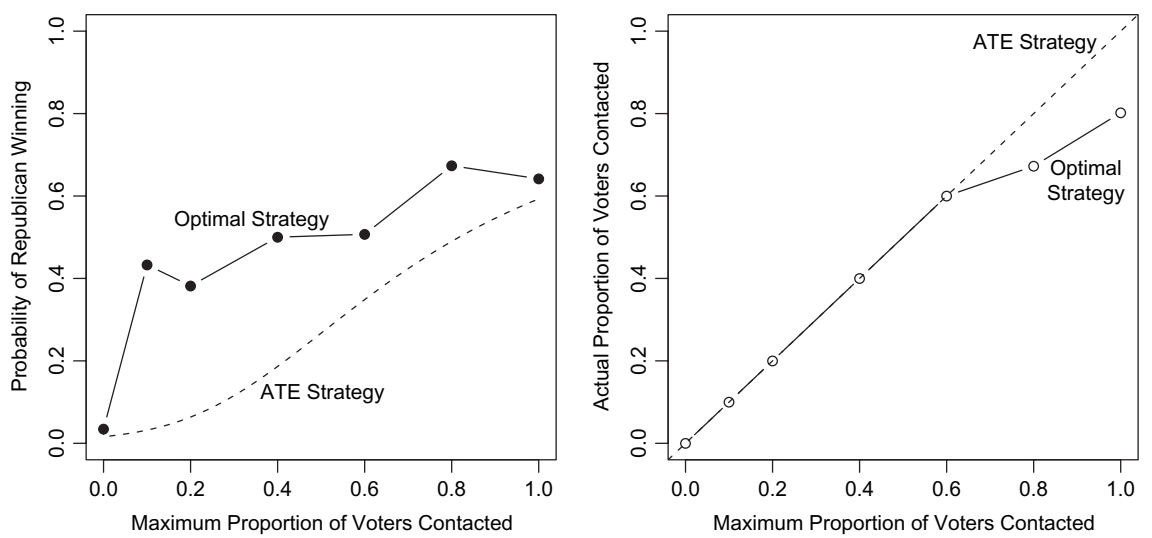

Fig. 5 Empirical evaluation of the performance of the proposed method for the partisan example. The results are calculated based on 10-fold cross validation. The left panel displays the estimated probability of the Republican candidate winning under two treatment strategies plotted over the maximum proportion of the electorate treatable under the budget constraint. The dashed lines represent the "ATE strategy" in which random voters are contacted. The solid lines represent the optimal strategy based on the methodology outlined in this paper, which uses covariate characteristics of voters to determine which voters receive the treatment. The optimal strategy outperforms the ATE approach strategy in most cases. The right panel plots the actual proportion of voters contacted by canvassing against the maximum proportion of voters contacted, which is determined by budget constraint. Only in the situations where nearly all voters can be contacted, does the algorithm choose not to canvass some voters.

optimal campaign strategy from the perspective of the Republicans. Indeed, an analysis from the Democratic perspective finds that the optimal strategy is to contact a very small percentage of voters. Available covariates we use are (from the voter file) gender, age, (from the survey) party identification, abortion views, senate vote, gubernatorial vote, and level of political interest.

Our algorithm finds two predictive variables (which also may be prescriptive), party identification and senate vote, and several purely prescriptive variables including political knowledge, political interest, and views on abortion. The exact combination of the prescriptive variables depends on the budget constraint. This variable selection is in line with the analysis of Arceneaux and Kolodny (2009) that finds heterogeneous treatment effects among low-interest Republicans as well as differential effects depending on prochoice/pro-life views. Figure 5 compares the optimal strategy with the ATE strategy in which randomly selected voters are contacted. The left panel shows that under the optimal strategy just contacting the $10 \%$ of the electorate increases the probability of Republican winning to about the same level as what could be achieved under the ATE strategy by contacting $70 \%$ of the electorate. The algorithm generally incrementally improves upon this result when the budget is increased so that a maximum of between $20 \%$ and $60 \%$ of the electorate can be contacted. Finally, the right panel shows that even if the partisan planner had the resources to contact every voter, some of the electorate is not worth contacting. All together, the analysis shows that the proposed algorithm allows partisan GOTV campaign planners to efficiently increase the probability of winning with limited resources.

\section{Concluding Remarks}

We have shown how to estimate heterogeneous treatment effects from randomized experiments and then use this information to derive an optimal policy. Although the statistical methods and algorithms used in this paper only represent one way of addressing the important methodological problem of treatment effect heterogeneity, we believe that the proposed framework will be useful as a general guidance for researchers and practitioners when thinking about how to get most information out of randomized experiments. Specifically, researchers can use statistical methods such as the one used in this paper to estimate heterogeneous treatment effects as a function of observable characteristics while avoiding the risk of overfitting. Then planners can use this information and derive, under a budget constraint, an optimal policy to determine which subgroups should receive which treatment. This can be done by defining an appropriate 
target population given the experimental sample and choosing an objective function while incorporating prior information whenever it exists.

We apply our method to the area of voter mobilization campaigns where political scientists have recently conducted over 100 randomized field experiments. We demonstrate how to exploit these experiments and derive an optimal campaign strategy for both nonpartisan and partisan campaigns. Interestingly, the problem of optimizing campaign tactics across voter characteristics was first introduced by Kramer (1966) more than 40 years ago. Our proposed methodology extends this pioneering work by placing the campaign planner's problem in the formal framework of statistical decision theory and applying the modern statistical methods. Clearly, many important issues are not addressed in this paper and remain to be studied. They include the question of how to extrapolate the results of an experiment to a different population and to a different election.

We conclude this paper by repeating a central point of our argument that the estimation of heterogeneous treatment effects can inform policy makers as well as academic researchers. In the aforementioned article, Kramer made the following observation:

In the past two decades, the use of quantitative methods as aids for decision-making has become common in many fields. [. . . ] By and large, however, these efforts have not been made by political scientists. [. . .] This is unfortunate, for many of the traditional concerns of political scientists appear to be quite susceptible to this sort of analysis. (137)

Despite this plea, political scientists have generally stayed away from the use of quantitative methods to directly inform policy makers who must make decisions using available data. However, the improvement in the quality of empirical studies, both experimental and observational, that have occurred over the last few decades in the discipline should present a great opportunity for current and future political scientists to take up Kramer's proposal (see, e.g., Lovett and Peress 2010).

\section{Funding}

National Science Foundation (SES-0752050); the Princeton University Committee on Research in the Humanities and Social Sciences.

\section{References}

Arceneaux, Kevin, and Robin Kolodny. 2009. Educating the least informed: Group endorsements in a grassroots campaign. American Journal of Political Science 53:755-70.

Berger, James O. 1985. Statistical decision theory and Bayesian analysis. 2nd ed. New York: Springer.

Breiman, Leo, Jerome Friedman, Charles J. Stone, and R. A. Olshen. 1984. Classification and regression trees. London: Chapman \& Hall.

Chipman, Hugh A., Edward I. George, and Robert E. McCulloch. 2010. BART: Bayesian additive regression trees. Annals of Applied Statistics 4:266-98.

Cole, Stephen R., and Elizabeth A. Stuart. 2010. Generalizing evidence from randomized clinical trials to target populations: The ACTG 320 trial. American Journal of Epidemiology 172:107-15.

Cox, David R. 1958. Planning of experiments. New York: John Wiley \& Sons.

Dale, Allison, and Aaron Strauss. 2008. Mobilizing the mobiles: How text messaging can boost turnout. In Politicking online: The transformation of election campaign communications, ed. Costas Panagopoulos, 152-62. New Brunswick, NJ: Rutgers University Press.

2009. Don't forget to vote: Text message reminders as a mobilization tool. American Journal of Political Science 53:787-804.

Dehejia, Rajeev. 2005. Program evaluation as a decision problem. Journal of Econometrics 125:141-73.

Druckman, James N., Donald P. Green, James H. Kuklinski, and Arthur Lupia. 2006. The growth and development of experimental research in political science. American Political Science Review 100:627-35.

Eldersveld, Samuel J. 1956. Experimental propaganda techniques and voting behavior. American Political Science Review 50: $154-65$.

Feller, Avi, and Chris C. Holmes. 2009. Beyond toplines: Heterogeneous treatment effects in randomized experiments. Technical report. Oxford, UK: University of Oxford.

Gerber, Alan S., and Donald P. Green. 2000. The effects of canvassing, telephone calls, and direct mail on voter turnout: A field experiment. American Political Science Review 94:653-63.

Gerber, Alan S., Donald P. Green, and Christopher W. Larimer. 2008. Social pressure and voter turnout: Evidence from a large-scale field experiment. American Political Science Review 102:33-48.

Gerber, Alan S., Donald P. Green, and Ron Shachar. 2003. Voting may be habit-forming: Evidence from a randomized field experiment. American Journal of Political Science 47:540-50. 
Gosnell, Harold F. 1927. Getting-out-the-vote: An experiment in the stimulation of voting. Chicago, IL: University of Chicago Press. Green, Donald P., and Alan S. Gerber. 2008. Get out the vote: How to increase voter turnout. 2nd ed. Washington, DC: Brookings Institution.

Green, Donald P., and Holder L. Kern. 2010a. Generalizing experimental results. The Annual Meeting of the American Political Science Association, Washington, DC.

. 2010b. Detecting heterogenous treatment effects in large-scale experiments using Bayesian additive regression trees. The Annual Summer Meeting of the Society of Political Methodology, University of Iowa.

Gunter, Lacey, Ji Zhu, and Susan Murphy. 2007. Variable selection for optimal decision making. Proceedings of the 11th Conference on Artificial Intelligence in Medicine AIME 2007, LNCS/LNAI 4594:149-54.

Hartman, Erin, Richard Grieve, and Jasjeet S. Sekhon. 2010. From SATE to PATT: The essential role of placebo test combining experimental and observational studies. The Annual Meeting of the American Political Science Association, Washington, DC.

Hartmann, George W. 1936. A field experiment on the comparative effectiveness of "emotional" and "rational" political leaflets in determining election results. Journal of Abnormal Psychology 31:99-114.

Hill, Jennifer L. Forthcoming. Bayesian nonparametric modeling for causal inference. Journal of Computational and Graphical Statistics doi:10.1198/jcgs.2010.08162.

Horiuchi, Yusaku, Kosuke Imai, and Naoko Taniguchi. 2007. Designing and analyzing randomized experiments: Application to a Japanese election survey experiment. American Journal of Political Science 51:669-87.

Kramer, G. H. 1966. A decision-theoretic analysis of a problem in political campaigning. In Mathematical applications in political science. Vol. II, ed. J. L. Bernd, 137-60. Dallas, TX: Southern Methodist University.

Lovett, Mitch, and Michael Peress. 2010. Targeting political advertising on television. Technical report. Rochester, NY: University of Rochester.

Manski, Charles F. 2005. Social choice with partial knowledge of treatment response. Princeton, NJ: Princeton University Press.

Morton, Rebecca B., and Kenneth C. Williams. 2010. Experimental political science and the study of causality: From nature to the lab. New York: Cambridge University Press.

Nickerson, David W. 2008. Is voting contagious?: Evidence from two field experiments. American Political Science Review 102:49-57.

Nickerson, David W., and Kevin Arceneaux. 2009. Who is mobilized to vote? A re-analysis of eleven randomized field experiments. American Journal of Political Science 53:1-16.

Pocock, Stuart J., Susan E. Assmann, Laura E. Enos, and Linda E. Kasten. 2002. Subgroup analysis, covariate adjustment and baseline comparisons in clinical trial reporting: current practice and problems. Statistics in Medicine 21:2917-30.

Ripley, Brian D. 1996. Pattern recognition and neural networks. Cambridge: Cambridge University Press.

Rubin, Donald B. 1990. Comments on "On the Application of Probability Theory to Agricultural Experiments. Essay on Principles. Section 9" by J. Splawa-Neyman translated from the Polish and edited by D.M. Dabrowska and T.P. Speed. Statistical Science $5: 472-80$.

Savage, L. J. 1951. The theory of statistical decision. Journal of the American Statistical Association 46:55-67.

Stoye, Jörg. 2009. Minimax regret treatment choice with finite samples. Journal of Econometrics 151:70-81.

Wald, Abraham. 1950. Statistical decision functions. New York: Wiley. 